\title{
Expression of CXCL13, a chemokine highly upregulated in germinal center T-helper cells, distinguishes angioimmunoblastic T-cell lymphoma from peripheral T-cell lymphoma, unspecified
}

\author{
Karen L Grogg ${ }^{1}$, Ayoma D Attygale ${ }^{2}$, William R Macon ${ }^{1}$, Ellen D Remstein ${ }^{1}$, Paul J Kurtin ${ }^{1}$
} and Ahmet Dogan ${ }^{1}$

${ }^{1}$ Department of Laboratory Medicine and Pathology, Mayo Clinic, Rochester, MN, USA and ${ }^{2}$ Department of Histopathology, University College, London, UK

\begin{abstract}
The germinal center T-helper cell has been proposed as the cell of origin for angioimmunoblastic T-cell lymphoma. Our recent report of expression of CXCL13, a chemokine critical for germinal center formation and one of the most highly upregulated genes in the germinal center T-helper cell subset, in the majority of angioimmunoblastic T-cell lymphoma cases, provided further support for this theory. To determine the specifity of this marker for angioimmunoblastic T-cell lymphoma, we evaluated CXCL13 expression in 26 nodal-based peripheral T-cell lymphomas and 14 lymph nodes showing paracortical lymphoid hyperplasia. No significant paracortical CXCL13 staining was seen in the reactive lymph nodes. By WHO classification criteria, 20 of the lymphoma cases were considered peripheral T-cell lymphoma, unspecified, and six were reclassified as angioimmunoblastic T-cell lymphoma after immunohistochemical detection of disorganized follicular dendritic cell meshworks. Combining the results of our studies, 31 of $\mathbf{3 5}$ angioimmunoblastic T-cell lymphoma cases (89\%) showed CXCL13 expression, in contrast to two out of 20 peripheral T-cell lymphoma, unspecified cases (10\%). The two peripheral T-cell lymphoma, unspecified cases that were positive for CXCL13 showed a Lennert lymphoma-like histology. While these cases did not meet all histologic criteria for angioimmunoblastic T-cell lymphoma, they did show an increase in EBV-positive B cells, suggesting they may be histologic variants of angioimmunoblastic T-cell lymphoma. In conclusion, CXCL13 expression is a distinctive feature of angioimmunoblastic T-cell lymphoma, providing further support for the germinal center T-helper cell as the cell of origin for this neoplasm. Given its specificity when compared to cases of peripheral T-cell lymphoma, unspecified as well as paracortical lymphoid hyperplasia, it may be a useful marker in the diagnosis of angioimmunoblastic T-cell lymphoma.
\end{abstract}

Modern Pathology (2006) 19, 1101-1107. doi:10.1038/modpathol.3800625; published online 5 May 2006

Keywords: angioimmunoblastic T-cell lymphoma; CXCL13; peripheral T-cell lymphoma unspecified; germinal center T-helper cell

Peripheral T-cell lymphomas comprise approximately $10 \%$ of all malignant lymphomas. ${ }^{1}$ Although great progress has been made in recent years defining important subcategories of peripheral Tcell lymphoma, the largest subcategory of peripheral T-cell lymphoma, unspecified remains heteroge-

Correspondence: Dr KL Grogg, MD, Department of Laboratory Medicine and Pathology, Mayo Clinic, 200 1st Street, SW, Rochester, MN 55905, USA.

E-mail: grogg.karen@mayo.edu

Received 4 January 2006; revised 10 April 2006; accepted 11 April 2006; published online 5 May 2006 neous. Cases within this subtype may show significant histologic overlap with angioimmunoblastic T-cell lymphoma. Recent studies have suggested that a defining feature of angioimmunoblastic T-cell lymphoma may be its origin from germinal center T-helper cells. ${ }^{2-4}$ Typically, the tumor cells of angioimmunoblastic T-cell lymphoma have a Thelper cell phenotype expressing CD3, CD4 and frequently CD10, similar to a subset of normal germinal center T-helper cells. ${ }^{3}$ In early lymph node involvement by angioimmunoblastic T-cell lymphoma, the neoplastic T cells preferentially occupy the B-cell follicles and immediate perifollicular area, 
suggesting that the follicle center microenvironment plays a role in tumor development. ${ }^{2,4,5}$

The expression of CXCL13 by angioimmunoblastic T-cell lymphoma, as documented in our recent publication, ${ }^{6}$ provides another piece of evidence linking the tumor cells to germinal center T-helper cells. CXCL13, a chemokine critical for B cell entry into germinal centers, ${ }^{7}$ was identified by Kim et $a l^{8}$ as one of the most highly upregulated genes in the germinal center T-helper cell subset. Kim et al also reported selective upregulation of several transcription factors in germinal center T-helper cells. One of these was Bcl-6, which like CXCL13, is overexpressed in angioimmunoblastic T-cell lymphoma $^{9}$ (A Dogan and AD Attygalle, unpublished data).

In order to determine the specificity of CXCL13 expression for angioimmunoblastic T-cell lymphoma, we compared the immunohistochemical staining pattern in angioimmunoblastic T-cell lymphoma as observed in our previous study with staining in cases of nodal-based peripheral T-cell lymphoma, unspecified and lymph nodes showing paracortical lymphoid hyperplasia.

\section{Materials and methods}

Fourteen paraffin-embedded lymph nodes showing paracortical lymphoid hyperplasia were included in the study. As part of a laboratory validation study, these specimens had been previously evaluated for clonal T-cell receptor gamma gene rearrangements by PCR analysis with negative results. Each case was stained with antibodies directed against CD10 (Novocastra, clone 56C6), and CXCL13 (R\&D Systems, Minneapolis, MN, clone 53610). The pattern of CD10 and CXCL13 staining in the reactive lymph nodes was compared to the pattern of staining seen in 25 (of 29) angioimmunoblastic T-cell lymphoma cases as reported in our prior publication. ${ }^{6}$

Archived paraffin-embedded tissues from 26 patients previously diagnosed with lymph nodebased peripheral T-cell lymphoma at the Mayo Clinic were studied. Many of these cases were diagnosed in an era preceding the current WHO Classification. The diagnosis had been made following immunophenotyping studies and in most cases, molecular genetic analysis of the T-cell receptor genes. For the current study, in each case paraffin sections were stained according to previously described techniques ${ }^{10}$ with antibodies directed against the following antigens: CD3 (Cell Marque, Hot Springs, AR, polyclonal), CD4 (Novocastra, Burlingame, CA, clone 4B12), CD8 (Dako, Carpinteria, CA, clone C8/144B), CD10 (Novocastra, clone 56C6), CD20 (Dako, clone L26), CD21 (Dako, clone 1F8) and CXCL13 (R\&D Systems, Minneapolis, MN, clone 53610). In situ hybridization studies for Epstein-Barr virus (EBV) were performed in selected cases using probes that recognize EBV- encoded RNA (Epstein-Barr virus encoded RNA) according to previously described methods. ${ }^{11}$

After review of the immunophenotypic studies, the histopathologic criteria of the WHO Classification were applied in each case. ${ }^{1}$ Staining of any significant subset of the neoplastic infiltrate for CD10 or CXCL13 was regarded as a positive result. The percentage of neoplastic cells that were positive for each antigen was estimated, after determining which cells appeared to be included in the neoplastic population by cytologic and/or immunophenotypic features.

The Mayo Foundation Institutional Review Board approved this study.

\section{Results}

The pattern of CXCL13 and CD10 staining in angioimmunoblastic T-cell lymphoma cases was compared to that seen in 14 lymph nodes showing paracortical lymphoid hyperplasia. CD10 staining in the reactive lymph nodes was predominantly confined to the germinal centers, where the CD10positive follicle center B cells were intermixed with scattered more intensely-staining lymphocytes that presumably represented germinal center T-helper cells. CXCL13 positive cells in normal and reactive lymph nodes were also concentrated in the germinal centers, including follicular dendritic cells, histiocytes, and rare small lymphocytes (Figure 1). Follicular dendritic cells showed cytoplasmic staining with accentuation around the nuclei and little or no staining in the dendritic processes. In contrast to the clusters of CXCL13-positive cells seen in a perivascular and perifollicular distribution in angioimmunoblastic T-cell lymphoma cases, the paracortex of reactive lymph nodes contained singly and evenly distributed CXCL13-positive cells, consisting of histiocytes and rare small lymphocytes that comprised $<1 \%$ of the cells. One of the paracortical hyperplasia specimens involving an axillary lymph node showed unusual small clusters (consisting of two or three cells) of CXCL13-positive cells adjacent to hyperplastic lymphoid follicles. Investigation into the clinical record indicated that this patient had been diagnosed with peripheral T-cell lymphoma in a cervical lymph node approximately 4 years prior and achieved clinical remission after CHOP chemotherapy. Although the axillary lymph node was not diagnostic of lymphoma given the lack of detectable clonal T-cell receptor gene rearrangements, the patient received additional chemotherapy for presumed lymphoma relapse. Three years subsequent to the nondiagnostic axillary lymph node specimen, the patient had documented relapse of peripheral T-cell lymphoma in inguinal lymph nodes. Review of the relapse specimen showed features characteristic of angioimmunoblastic T-cell lymphoma, with proliferation of follicular dendritic cell meshworks and EBV-positive B immunoblasts. Additional material 

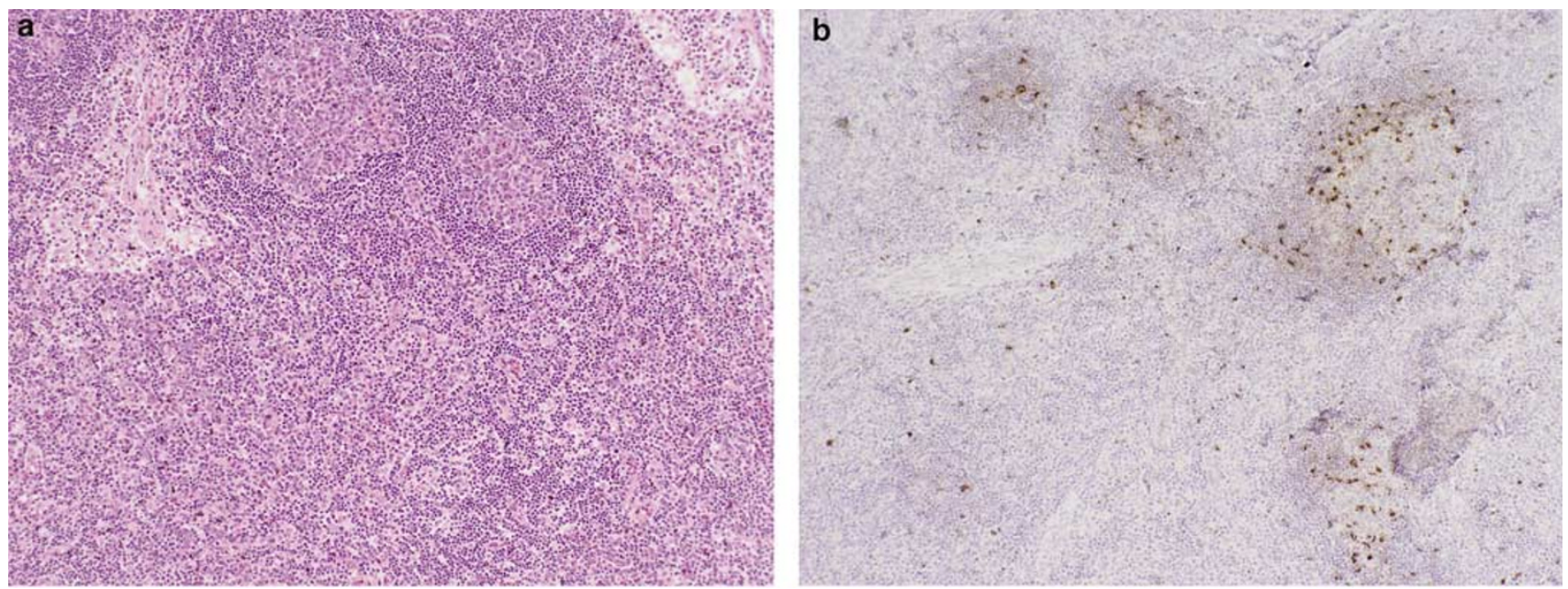

Figure 1 Expression pattern of CXCL13 in reactive lymph nodes. (a) The lymph node shows follicular and paracortical hyperplasia (hematoxylin and eosin). (b) Immunoperoxidase studies for CXCL13 show positive cells confined mostly to the germinal center, including follicular dendritic cells, small lymphocytes, and histiocytes. The paracortex contains rare, singly distributed CXCL13-positive cells.

from the inguinal lymph nodes for CXCL13 staining was not available.

According to the WHO classification, the diagnosis of angioimmunoblastic T-cell lymphoma rests on morphologic criteria, which includes a polymorphous infiltrate in the lymph node paracortex with a prominent proliferation of high endothelial venules and follicular dendritic cells. ${ }^{1}$ Using these criteria, six of the 26 peripheral T-cell lymphoma cases were reclassified as angioimmunoblastic T-cell lymphoma after immunohistochemical demonstration of a disorganized proliferation of CD21-positive follicular dendritic cell meshworks. Of the reclassified cases, all six showed cytoplasmic staining for CXCL13 in cells that corresponded to the CD3, CD4 and CD10 (positive in four of six cases) positive tumor cells (Figure 2). Combined with our previously published results, CXCL13 expression was seen in the tumor cells in 31 of 35 cases of angioimmunoblastic T-cell lymphoma $(89 \%)$, and CD10 expression was seen in 26 cases (74\%) (Table 1). The CXCL13 staining highlighted the clustering of atypical cells in a perivascular distribution in cases with diffuse lymph node effacement (pattern III as described by Attygalle ${ }^{2}$ ), and their presence as large aggregates of cells at the borders of hyperplastic or regressed lymphoid follicles in cases with partially retained lymph node architecture (patterns I and II as described by Attygalle ${ }^{2}$ ). Although the polymorphous nature of the infiltrate made definite identification of the tumor cells difficult in some cases, CXCL13 appeared to stain the majority of the T cells (estimated at $80-90 \%$ in the majority of cases, range $40-90 \%$ ) with uniform strong intensity, compared to the more variable and sometimes weak intensity of CD10 staining in the T-cell population (estimated at 50$60 \%$ in the majority of cases, range $20-90 \%$ ). One particularly interesting case showed classic features of angioimmunoblastic T-cell lymphoma in one half of the lymph node, with the other half showing transformation to an EBV-positive large B-cell lymphoma. The concentration of CXCL13 and CD10-positive cells was most intense surrounding and infiltrating the sheets of EBV-positive B cells. Disorganized follicular dendritic cell meshworks were also present in those areas.

In all, 20 cases were confirmed as peripheral Tcell lymphoma, unspecified based on WHO criteria that require effacement of normal lymph node architecture by a diffuse infiltrate, usually polymorphous in composition, that includes a medium or large-sized T-cell population showing a cytological spectrum. ${ }^{1}$ By definition, these cases did not fit into any other recognizable subtype of T-cell neoplasia. In most cases (15 out of 20), clonal T-cell receptor gene rearrangements had been demonstrated by PCR and/or Southern blot analysis. The cases included 15 cases that expressed CD4, two that expressed CD8, two that were negative for CD4 and CD8, and one that coexpressed CD4 and CD8. In contrast to the angioimmunoblastic T-cell lymphoma cases, CXCL13 expression by the T cells was observed in only two out of 20 peripheral T-cell lymphoma, unspecified cases $(10 \%)$. Neither of these cases showed positivity for CD10, and follicular dendritic cell proliferation could not be demonstrated immunohistochemically with stains for CD21. A single case of peripheral T-cell lymphoma, unspecified showed positivity for CD10, but lacked expression of CXCL13 and did not show follicular dendritic cell proliferation.

The two cases of peripheral T-cell lymphoma, unspecified that were CXCL13 positive showed a Lennert lymphoma-like histology, with an extensive histiocytic population intermingled with the tumor 

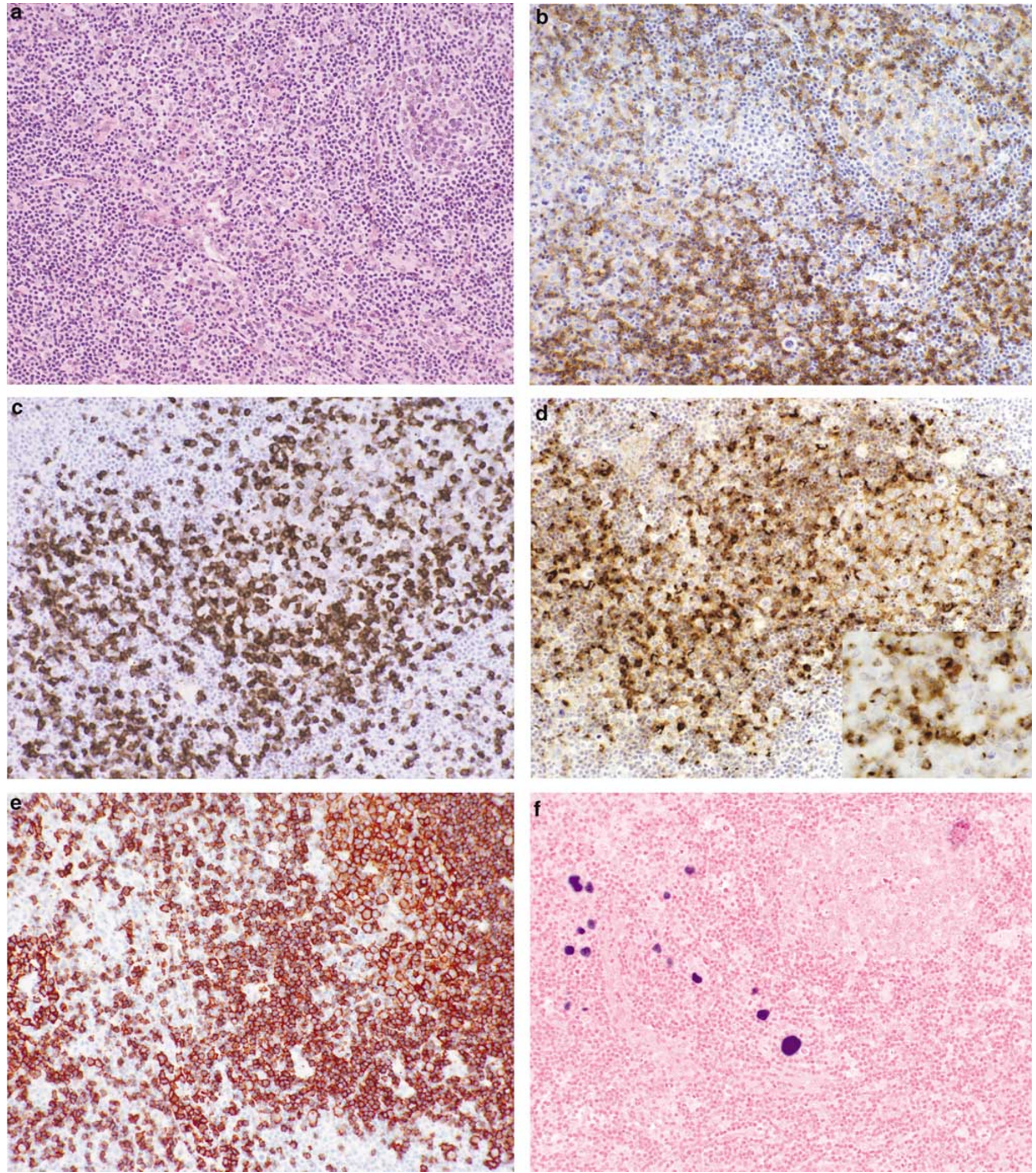

Figure 2 Angioimmunoblastic T-cell lymphoma expresses CXCL13. (a) A polymorphous paracortical infiltrate is seen in this case of angioimmunoblastic T-cell lymphoma (hematoxylin and eosin). (b) CD4-positive neoplastic T cells are clustered in the perifollicular area and extend into the paracortex (immunoperoxidase). (c) The neoplastic T cells show intense CD10 positivity (immunoperoxidase). (d) Strong cytoplasmic staining for CXCL13 is seen in the same cell population (inset, immunoperoxidase). (e) Increased numbers of CD20positive B cells and immunoblasts are present in the paracortex (immunoperoxidase). (f) Immunoblasts are positive for EBV (in situ hybridization).

cells (Figure 3). In one case the neoplastic cells were CD3 and CD4-positive; the other case showed an aberrant T-cell phenotype with immunohistochem- ical positivity for CD2 and CD7, and negativity for CD3, CD4, CD5, CD8, CD30 and beta F1. A distinct clonal T-cell receptor gene rearrangement was 
Table 1 Proportion of AITL and PTCL-u cases expressing CD10 and CXCL13 as assessed by immunohistochemistry

\begin{tabular}{lcc}
\hline Lymphoma subtype & CD10 positive & CXCL13 positive \\
\hline AITL $(n=35)^{\mathrm{a}}$ & $26(74 \%)$ & $31(89 \%)$ \\
PTCL-u $(n=20)$ & $1(5 \%)$ & $2(10 \%)$
\end{tabular}

AITL, angioimmunoblastic T-cell lymphoma; PTCL-u, peripheral T-cell lymphoma, unspecified.

${ }^{\mathrm{a}}$ Includes combined results of current and previous study. ${ }^{6}$

detected by PCR and Southern blot analysis. Although these cases were not classified as angioimmunoblastic T-cell lymphoma because of the lack of defining histologic criteria, namely follicular dendritic cell proliferation, they possessed some features that were similar to angioimmunoblastic T-cell lymphoma. Both cases displayed significantly increased numbers of EBV-positive cells and scattered B immunoblasts as is characteristic of angioimmunoblastic T-cell lymphoma, and in focal areas assumed a more polymorphous composition with proliferation of high endothelial venules. The peripheral T-cell lymphoma, unspecified case that showed CD10 positivity but lacked CXCL13 expression and follicular dendritic cell proliferation also showed an increase in the number of EBV positive cells, although fewer in number compared to the other 2 cases.

\section{Discussion}

Peripheral T-cell lymphomas often show overlapping histologic features, and also share the tendency for systemic symptomatology related to cytokine production. ${ }^{1}$ Differential expression of chemokines and their receptors has in fact been associated with certain peripheral T-cell lymphoma subtypes. A recent DNA microarray study documented CXCL13 expression in angioimmunoblastic T-cell lymphoma, and a minor subset of other peripheral T-cell lymphomas. ${ }^{12}$ The results of this and our previous study confirm expression of the chemokine CXCL13 within the $\mathrm{T}$ cells of angioimmunoblastic T-cell lymphoma, and also show that this feature distinguishes it from other nodal peripheral T-cell lymphomas as well as from lymph nodes showing paracortical lymphoid hyperplasia. As it has been shown that CXCL13 is differentially expressed in germinal center T-helper cells compared to other CD4-positive T-cell subsets, ${ }^{8}$ this finding adds to the growing body of evidence that the germinal center T-helper cell is the cell of origin for angioimmunoblastic T-cell lymphoma.

By considering the role that CXCL13 plays within normal lymphoid follicles, one can gain insight into the histologic patterns seen in angioimmunoblastic T-cell lymphoma. During a normal immune response, activated germinal center T-helper cells express high levels of CXCL13, which induces chemotaxis of cells with upregulated expression of the CXCL13 receptor CXCR5, including IgD-positive $\mathrm{B}$ cells and certain T-helper cell subsets. ${ }^{7,13}$ This results in B cell entry into the follicle, as well as relocalization of unactivated germinal center T-helper cells and central memory $\mathrm{T}$ cells from the paracortex toward the follicles, where they can provide help to B cells during the process of antigen affinity maturation and isotype switching. CXCL13 also induces B cells to upregulate lymphotoxinalpha, which itself promotes CXCL13 expression in follicular dendritic cells, establishing a positive feedback loop thought to be critical in the organization of the lymphoid follicle. ${ }^{13}$

CXCL13 expression in angioimmunoblastic T-cell lymphoma provides explanation for the composition and distribution of cells in the neoplastic infiltrate. The pivotal role that CXCL13 plays in the organization of the lymphoid follicle may explain the localization of the neoplastic $\mathrm{T}$ cells to the follicular and perifollicular areas. The intimate association of the tumor cells with follicular dendritic cell meshworks and the clustering of tumor cells visualized on CXCL13-stained sections may be related to the expression of both CXCL13 and its receptor by the germinal center T-helper cell subset. Given that a microenvironment rich in CXCL13 would recruit additional, potentially nonneoplastic, germinal center T-helper cells, it is possible that only a subset of the CXCL13 positive cell population represents the neoplastic clone. The proliferation of B cells in angioimmunoblastic T-cell lymphoma, seen as follicular hyperplasia, and in later stages a more immunoblastic and plasmacytic expansion, could be explained by the physiologic role that CXCL13 plays in B cell recruitment and maturation. One could also postulate that the expansion of EBV positive cells within the neoplastic infiltrate could potentially be due to recruitment of EBV infected B cells with elevated levels of CXCR5 expression. Further studies are needed to confirm this hypothesis. The expanded follicular dendritic cell meshworks characteristic of angioimmunoblastic T-cell lymphoma should be expected given that induction and proliferation of follicular dendritic cells is a downstream effect of CXCL13 secretion. ${ }^{7}$

Identification of CXCL13 expression as a distinctive feature of angioimmunoblastic T-cell lymphoma may help to redefine this subtype of peripheral T-cell lymphoma, through recognition of histologic variants. CXCL13 expression was seen in two out of 20 cases $(10 \%)$ of peripheral T-cell lymphoma, unspecified, similar to the $11 \%$ identified by DNA microarray study and reported by Ohshima et al. ${ }^{12}$ The two cases in our study displayed some of the histologic features of angioimmunoblastic T-cell lymphoma, but lacked proliferation of follicular dendritic cells. One case showed very limited expression of T-cell antigens, which would be very 

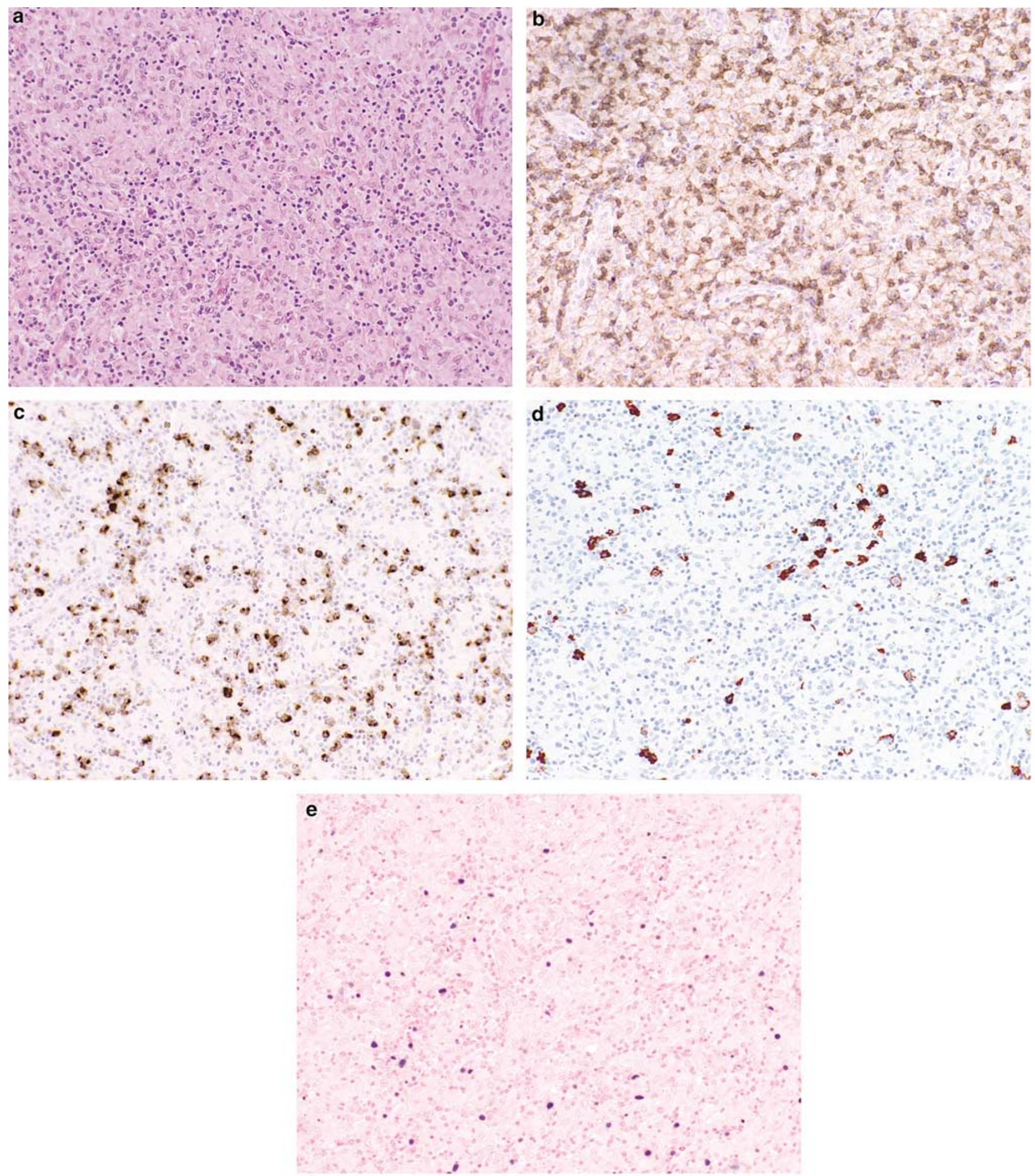

Figure 3 An example of peripheral T-cell lymphoma, unspecified that expresses CXCL13. (a) The lymph node biopsy shows Lennert lymphoma-like histologic features, with an extensive histiocytic infiltrate (hematoxylin and eosin). (b) A stain for CD4 shows intense membrane staining in the neoplastic T cells, and weaker staining in the reactive histiocytes (immunoperoxidase). (c) Strong CXCL13 expression is seen in a cell population that corresponds to the T cells (immunoperoxidase). (d) Features similar to angioimmunoblastic Tcell lymphoma include increased numbers of CD20-positive B cells, showing a cytologic spectrum from small lymphocytes to large immunoblasts (immunoperoxidase). (e) Increased numbers of EBV positive cells are also present (in situ hybridization).

unusual for angioimmunoblastic T-cell lymphoma. Interestingly, both cases featured a Lennert lymphoma-like histology, with numerous infiltrating epithe- lioid histiocytes. This may suggest that some of the cases historically described as Lennert lymphoma represent a histologic variant of angioimmunoblastic 
T-cell lymphoma, in which follicular dendritic cell proliferation is less pronounced. In fact, Lennert himself identified a subset of peripheral T-cell lymphoma cases with a high content of epithelioid cells that had clinical and morphologic characteristics of angioimmunoblastic T-cell lymphoma. ${ }^{14}$

CXCL13 expression provides another diagnostic marker for angioimmunoblastic T-cell lymphoma, which appears to have slightly better overall sensitivity and more uniform and intense positivity when compared to CD10, staining the majority of CD4 positive $\mathrm{T}$ cells in the neoplastic infiltrate. CXCL13 expression may also be useful to recognize very early lymph node involvement by angioimmunoblastic T-cell lymphoma. We observed that the clustering of CXCL13-positive cells in the paracortex as seen in angioimmunoblastic T-cell lymphoma is not commonly seen in lymph nodes showing paracortical lymphoid hyperplasia. The case of lymphoid hyperplasia, in which, we noted a slight increase in clustering of paracortical CXCL13-positive cells is intriguing, in that it may indicate an early lesion of angioimmunoblastic T-cell lymphoma, given the history and subsequent relapse of angioimmunoblastic T-cell lymphoma in that patient.

In addition to being a sensitive marker to add to the diagnostic armamentarium for angioimmunoblastic T-cell lymphoma, expanding our knowledge of the normal cellular counterpart of this peripheral T-cell lymphoma subtype may lead to new treatment strategies. Patients with angioimmunoblastic T-cell lymphoma encounter clinical effects of marked dysregulation of the immune system rather than direct complications of tumor growth, and there have been attempts to moderate these effects using immunomodulatory approaches. ${ }^{15,16}$ The information gained by studying normal germinal center T-helper cells may be helpful in the development of targeted immunomodulatory treatment strategies for this otherwise fatal disease.

\section{Acknowledgements}

Research supported in part by the University of Iowa/Mayo Clinic Lymphoma Specialized Program of Research Excellence (SPORE P50 CA97274).

\section{References}

1 Jaffe E, Ralfkiaer E. WHO histological classification of mature T-cell and NK-cell neoplasms. In: Jaffe E, Harris $\mathrm{N}$, Stein $\mathrm{H}$ and Vardiman J (eds). World Health Organization Classification of Tumours. Pathology and Genetics of Tumours of Haematopoietic and Lymphoid Tissues. IARC Press: Lyon, France, 2001, pp 190-235.

2 Attygalle A, Al-Jehani R, Diss T, et al. Neoplastic T cells in angioimmunoblastic T-cell lymphoma express CD10. Blood 2002;99:627-633.

3 Dogan A, Attygalle A, Kyriakou C. Angioimmunoblastic T-cell lymphoma. Br J Haematol 2003;121:681-691.

4 de Leval L, Savilo E, Longtine J, et al. Peripheral T-cell lymphoma with follicular involvement and a CD4/ bcl-6+ phenotype. Am J Surg Pathol 2001;25:395-400.

5 Ree H, Kadin M, Kikuchi M, et al. Angioimmunoblastic lymphoma (AILD type T-cell lymphoma) with hyperplastic germinal centers. Am J Surg Pathol 1998; 22:643-655.

6 Grogg K, Attygalle A, Macon W, et al. Angioimmunoblastic T-cell lymphoma: a neoplasm of germinal center T helper cells? Blood 2005;106:1501-1502.

7 Ansel K, Ngo V, Hyman P, et al. A chemokine-driven positive feedback loop organizes lymphoid follicles. Nature 2000;406:309-314.

$8 \mathrm{Kim} \mathrm{C}$, Lim H, Kim J, et al. Unique gene expression program of human germinal center $\mathrm{T}$ helper cells. Blood 2004;104:1952-1960.

9 Ree H, Kadin M, Kikuchi M, et al. Bcl-6 expression in reactive follicular hyperplasia, follicular lymphoma, and angioimmunoblastic T-cell lymphoma with hyperplastic germinal centers: heterogeneity of intrafollicular T-cells and their altered distribution in the pathogenesis of angioimmunoblastic T-cell lymphoma. Hum Pathol 1999;30:403-411.

10 Kurtin P, Hobday K, Ziesmer S. Demonstration of distinct antigenic profiles of small B-cell lymphomas by paraffin section immunohistochemistry. Am J Clin Pathol 1999;112:319-329.

11 Chang K, Chen Y, Shibata D. Description of an in situ hybridization methodology for detection of EpsteinBarr virus RNA in paraffin-embedded tissues, with a survey of normal and neoplastic tissues. Diagn Mol Pathol 1992;1:246-255.

12 Ohshima K, Karube K, Kawano R, et al. Classification of distinct types of peripheral T-cell lymphoma unspecified, identified by chemokine and chemokine receptor expression: Analysis of prognosis. Int J Oncol 2004;25:605-613.

13 Ebert L, Schaerli P, Moser B. Chemokine-mediated control of $\mathrm{T}$ cell traffic in lymphoid and peripheral tissues. Mol Immunol 2005;42:799-809.

14 Patsouris E, Noel H, Lennert K. Angioimmunoblastic lymphadenopathy-type of $\mathrm{T}$ cell lymphoma with a high content of epithelioid cells. Histopathology and comparison with lymphoepithelioid cell lymphoma. Am J Surg Pathol 1989;13:262-275.

15 Dogan A, Ngu L, Ng S, et al. Pathology and clinical features of angioimmunoblastic T-cell lymphoma after successful treatment with thalidomide. Leukemia 2005;19:873-875.

16 Advani R, Warnke R, Sikic B, et al. Treatment of angioimmunoblastic T-cell lymphoma with cyclosporine. Ann Oncol 1997;8:601-603. 